\title{
A DNA inducing VLP vaccine designed for HIV and tested in mice
}

\author{
Alexandre Calazans $^{1,2 *}$, Cesar Boggiano ${ }^{1}$, Ross Lindsay ${ }^{1}$
}

1 Design and Development Laboratory, International AIDS Vaccine Initiative, Brooklyn, NY, United States of America, 2 Center for Technological Development in Health, Oswaldo Cruz, Foundation, Rio de Janeiro, Brazil

*acalazans@cdts.fiocruz.br

\section{Abstract}

We developed a DNA vaccine that induces the formation of a VLP in vivo. This VLP was designed to elicit neutralizing antibodies, to induce better T-cell responses and to activate the innate immune system. Overall, 5 groups of 10 mice were electroporated with the following constructs: pVLP-LTR-GagPro [full], pVLP-GagPro [VLP wihout RNA], pVLP-LTR-Gag [VLP immature], pVLP-Gag and pVLP-EnvBG505 [regular DNA vaccine] and a mock group. We performed ICS on the mouse spleens and performed ELISA for ENV antibodies and a Luminex assay for inflammatory cytokines. The VLP showed good binding to the neutralizing antibodies. The percentage of CD4 cells producing cytokines was $0.1 \%$ [IFNg], $0.15 \%$ [IL-2] and $0.2 \%$ [TNFa] for the construct pVLP-LTR-GagPro. The percentage of CD8 cells producing cytokines was $0.3 \%[\mathrm{IFNg}], 0.2 \%[\mathrm{IL}-2]$ and $0.25 \%[\mathrm{TNFa}]$. All pVLP constructs induced more antibodies for EnvBG505 than the regular DNA vaccine Env. The pVLP-LTRGagPro induced more IL-1B than the other constructs 24 hours post-vaccination.

\section{pone. 0183803}

Editor: Shixia Wang, University of Massachusetts Medical School, UNITED STATES

Received: April 17, 2017

Accepted: August 13, 2017

Published: August 24, 2017

Copyright: This is an open access article, free of all copyright, and may be freely reproduced, distributed, transmitted, modified, built upon, or otherwise used by anyone for any lawful purpose. The work is made available under the Creative Commons CCO public domain dedication.

Data Availability Statement: All relevant data are within the paper and its Supporting Information files.

Funding: This work was supported by a Gilead Foundation Fellowship Grant to Alexandre Calazans.

Competing interests: The authors have declared that no competing interests exist.

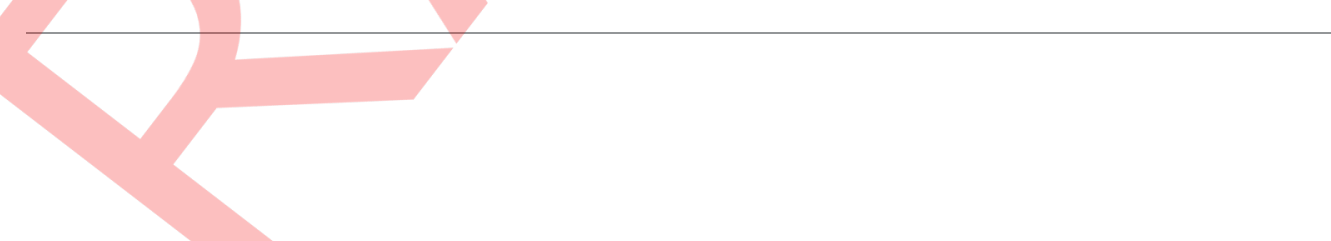

\section{Introduction}

It has been more than 25 years since HIV-1 was identified as the causative agent for AIDS [1] [2]. More than 60 million people worldwide have been infected with HIV-1, mostly in the developing world, and nearly half of these individuals have died. The development of a safe and effective HIV-1 vaccine would undoubtedly be the best solution for the ultimate control of the worldwide AIDS pandemic. Unfortunately, HIV-1 vaccine development efforts have not yet proven successful. The extraordinary diversity of HIV-1, the capacity of the virus to evade adaptive immune responses, the inability to induce broadly reactive antibody responses, the early establishment of latent viral reservoirs, and the lack of clear immune correlates of protection represent unprecedented challenges for vaccine development [3]. The main goal of an HIV-1 vaccine would be either to prevent infection or to reduce viral loads after infection. A perfect vaccine would stop infection and provide sterilizing immunity. In contrast, a big number of licensed viral vaccines work by controlling subclinical viral replication and by preventing clinical disease. Such partial control, as exemplified by a decline in peak and setpoint viral 
loads following infection, has been showed in certain preclinical studies by vaccines that elicit T-cells responses [4].

As mentioned above, $\mathrm{T}$ cell responses are desired to be elicited by an effective HIV vaccine [5]. It is well known that Gag CD8 $\mathrm{T}$ cell responses are associated with lowering viremia, while Env CD8 T-cells are related to higher viremia [6]. Antibodies that neutralize diverse strains of HIV-1 develop in approximately 20\% of HIV-1-infected individuals [7]. The RV144 trial demonstrated a $31 \%$ vaccine efficacy at preventing HIV-1 infection. Antibodies against the HIV-1 envelope variable loops 1 and 2 [Env V1 and V2] correlated inversely with infection risk [8]. New adjuvants and delivery systems are also necessary in new vaccines for HIV. They have been designed based upon their capacity to stimulate innate immune sensors and target antigens to dendritic cells, the cells responsible for initiating adaptive immune responses. Including these adjuvants and delivery systems into vaccines can positively alter the nature of the adaptive immune response, resulting in augmented protection [9]. For example, innate immune responses modulate $\mathrm{T}$-cell differentiation and control the antibody response at critical checkpoints of antigen-driven B cell differentiation. Viruses are primarily detected by the innate immune system through recognition of viral nucleic acids. Genomic HIV-1 RNA induces innate responses through Toll-like receptor 7 (TLR-7) and through retinoic acid inducible gene RIG-I-like receptor sensing of secondary-structured RNA [10].

Traditionally, vaccine development involves tradeoffs between immunogenicity and safety. Live-attenuated vaccines typically offer rapid and durable immunity but have reduced safety when compared to inactivated vaccines. In contrast, the inability of inactivated vaccines to replicate enhances safety at the expense of immunogenicity, often necessitating multiple doses and boosters. As the VLP formed by DNA vaccination is similar to the HIV virus itself, we think this vaccine can work as a live vaccine, but inactivated. In other words, the vaccine will be non-replicative. Actually, will have one cycle of replication. Once the VLP is formed, it will infect a cell and its RNA will be translated into new proteins that will form a new VLP which will not contain RNA. This will result in the augmented expression of vaccine antigens in vivo. Thus this VLP of HIV can use the host-cell protein processing pathways that lead to antigen presentation via the major histocompatibility complex class I and consequent cytotoxic T-cell stimulation [11].

Based on the above facts, we propose the development of a new HIV-1 DNA vaccine candidate, which is designed to elicit neutralizing antibodies [NAb] and T-cell responses concomitant with HIV-1 specific innate responses. Our approach is to develop a dual promoter DNA vaccine vector. The rationale is to have the DNA vaccine driving in vivo assembly and budding of a mature Gag VLP containing BG505 Env and also carrying a portion of the HIV-1 genome. The idea is not to use VLP, but rather the DNA itself, as vaccine to drive the assembly and budding of the VLP in vivo. One promoter drives the expression of HIV-1 LTR-GagPro composed of HIV nucleic acid sequences, which can encode mature Gag proteins, while the LTR-GapPro RNA will be encapsulated in the particles due the presence of the Psi sequence in the LTR. This viral RNA in the VLP can trigger the innate immune system through ligation to TLR-7 and RIG-1. The other promoter expresses Rev and Env BG505. Rev protein allows correct HIV RNA processing and packaging, and Env will be incorporated into the budding VLP. The membrane of a virus, here a VLP, is the ideal place for the right conformation of the HIV envelope. It is the place where envelope exposes its CD4 binding sites, essential for neutralizing antibodies to bind. We tested this new DNA vaccine approach in mice and produced good immunogenicity results. The vaccine induced high levels of $\mathrm{T}$ cell responses, good titers of Env specific antibodies and was able to induce IL-1 $\beta 24$ hours post-vaccination, suggesting innate immune system activation. 


\section{Methods}

\section{Sequences and gene synthesis}

All genes orders were made to GeneArt $₫$ and were human optimized. The LTRGagPro was synthetized as the first 1925 nucleotides of the prototype HIV-1 HXB2 genome. The Rev2AEnvBG505 was ordered as the Rev gene followed by a furin site (RKRR), the $2 \mathrm{~A}$ sequence of Tomato aspermy virus (TAV) and the Env BG505 gene [12]. The native signal sequence of the HIV-1 envelope was replaced by a CD5 signal sequence.

\section{Cloning}

We built the pCMV-dualpromoter vector as a bi-cistronic eukaryotic expression vector with 2 CMV promoters and kanamycin resistance, to be a DNA vaccine vector. We cloned the LTRG agPro sequence in the BamH1 and EcoRV sites of the MCS1 and cloned the Rev2AEnvBG505 in the EcoRI and XbaI sites of the MCS2. We called the plasmid formed pVLP-LTRGagPro. The others construct, pVLP-GagPro, pVLP-LTRGag, pCMV-Gag and pCMV-Env, were built by PCR amplification of the target region and cloning of the MCS1 (Fig 1). Rev2aEnvBG505 retained the same GagPro and LTRGag. pCMV-Gag and-Env contained only these genes. All plasmids were prepared prior vaccination with HiSpeed Plasmid Mega EF Kit [Qiagen, Germany] an Endo-free plasmid purification kit.

\section{Western blot}

To evaluate the processing of Gag proteins in the VLP, the LTR-GagPro [processed Gag] and LTR-Gag [non-processed Gag] plasmids were transfected to 293-T cells with $1.0 \mu \mathrm{g}$ of DNA in 12 a well plate, using the reagent Lipofectamine $(2000$ [Invitrogen, USA]. 48 hours after the initiation of transfection, cells were removed and lysed in the presence of a low salinity buffer

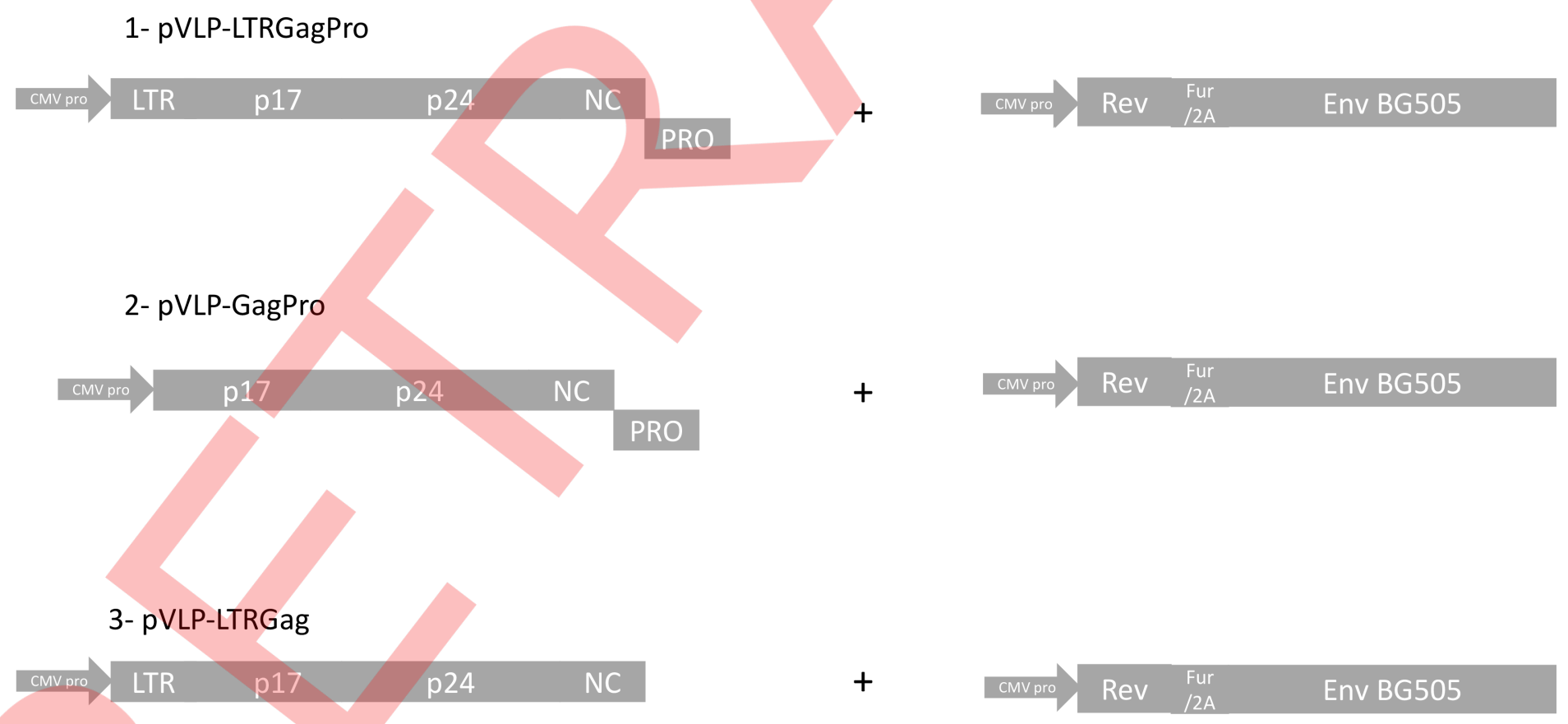

Fig 1. The HIV constructions used in this work. 1- pVLP-LTRGagPro, 2- pVLPGagPro, 3- pVLP-LTRGag; associated with Rev2AEnvBG505, the construction that carries the HIV-1 subtype A Envelope BG505 and the gene Rev, necessary for RNA packaging. The CMV promoters [CMV pro] location are shown. 
[10 mM Tris-Cl pH 7.5, 5 mM EDTA pH 8.0, $150 \mathrm{mM} \mathrm{NaCl}, 0.1 \% \mathrm{NP}-40]$ at $4^{\circ} \mathrm{C}$ for $30 \mathrm{~min}-$ utes. The lysate was clarified by centrifugation at $13,000 \mathrm{~g}$. The supernatant was collected, filtered $(0.22 \mu \mathrm{M}$ membrane, Millipore) and concentrated in a $20 \%$ sucrose buffer in an ultracentrifuge ( $44000 \mathrm{~g} / 45$ minutes $/ 4^{\circ} \mathrm{C}$ ). Then the supernatant was discarded and the VLP was resuspended in $100 \mu \mathrm{L}$ of PBS.

Proteins were separated by $12.5 \%$ SDS-PAGE and then transferred to nitrocellulose membranes (Hybond-ECL, GE Healthcares, USA) using the BIORAD transfer mini-vessel. The transfer was carried out in transfer buffer (20\% Tris-glycine 5X, 20\% absolute methanol) for 16 hours at 20 Volts. After transfer, the membranes were incubated at room temperature for 2 hours in blocking buffer (1\% PBS, 5\% skim milk, 0.2\% tween-20, 0.5\% BSA). Then, the primary antibody (Polyclonal Anti-p24GagHIV Antibody) was added and incubated for 2 hours at room temperature. At the end of this period, the membranes were washed 3 times with $1 \mathrm{X}$ PBS containing $0.2 \%$ Tween-20. The secondary antibody was added in the same buffer (antimouse immunoglobulins) and incubated for 1 hour at room temperature.

Finally, the membranes were washed 5 times with PBS-Tween and developed by chemiluminescence using the reagent kit for ECL protein detection (GE Healthcare, USA) according to the manufacturer's guidelines.

Pull-down, ELISA and PCR: We transfected the pVLP-LTRGagPro into 293-T cells and collected the supernatant. Then we labelled the VLP in the supernatant with biotinylated (OneStep Biotinylation kit, Miltenyi, Germany) neutralizing antibodies (b12, PG9, PG16, PGT126, PGT145 and PGV04). Then we pulled down the labelled VLP with Anti-Biotin Microbeads (Miltenyi).

We performed an HIV-1 p24 ELISA (ZeptoMetrix, USA) with the purified VLP. Additionally, we extracted the viral RNA of the purified VLP with the QIAamp Viral RNA Mini Kit (Qiagen, Germany) and made cDNA with the High Capacity cDNA kit (ThermoFisher Scientific, USA). We then performed PCR for the Gag region.

\section{Vaccination in mice}

We tested this vaccine in mice, and 10 animals were used for each group of vaccine tested. $\mathrm{Balb} / \mathrm{c}$ animals were used. The groups were pVLP-LTRGagPro, pVLP-GagPro, pVLPLTRGag, pCMV-Gag + pCMV-Env, and an empty vector. The animals were vaccinated by electroporation (TriGrid System, USA) with $20 \mu \mathrm{g}$ of DNA, twice, every 3 weeks. Serum was collected 24 hours after the second exposure and during week 5 . On week 9 , animals were bled for serum then euthanized, and the spleens were removed for $\mathrm{T}$ cell analysis. The mice will be euthanized via $\mathrm{CO}_{2}$ inhalation or Euthasol $(100 \mathrm{mg} / \mathrm{kg})$ administered IP and a terminal blood collection via cardiac puncture was performed. The condition of the animals was monitored on daily basis. No animals showed signs of illness post treatment. And no animal died (without euthanasia) due to the experimental procedures. An IACUC protocol (IBC-13-012) was issued, for this study at the State University of New York Downstate Medical Center, where IAVI houses the animal incubators. Institutional Care and Use Committee (IACUC) specifically approved this study.

\section{Env BG505 ELISA}

Serial dilutions of heat inactivated sera from week 5 and 9 were incubated for $1 \mathrm{hr}$ on 96 -well plates which were previously coated with Env BG505 protein [homemade produced], then blocked for 1 hour with 3\% BSA/PBS. Plates were washed in 0.05\% PBS/Tween and incubated for $1 \mathrm{hr}$ with the alkaline 236 phosphatase-conjugated secondary antibodies. After washing, plates were incubated for $30 \mathrm{~min}$ with nitrophenylphosphate and optical density was measured 
at $450 \mathrm{~nm}$ using SoftMAx Pro GxP v5 (Molecular Devices, US). All data shown have the 'no sera' background signal subtracted.

\section{Intracellular cytokine staining [ICS]}

PBMC between 600,000 and 1,000,000 were incubated with gag protein and HIV-1 Consensus B Gag peptide pools (The following reagent was obtained through the NIH AIDS Reagent Program, Division of AIDS, NIAID, NIH: HIV-1 Con B Gag Peptide Pool [cat \#12425]) or gp 120 protein for 9 hours with brefeldin A (Sigma, US), CD49d, and CD28 (BD Biosciences, US) at $37^{\circ} \mathrm{C}$ with $5 \% \mathrm{CO}_{2}$. Cells were washed and stained with LIVE/DEAD -Aqua Viability fixable cell stain (Invitrogen, US), then washed and stained for extracellular markers with CD3-Pacific Blue, CD4-PerCP Cy5.5, and CD95-APC [BD Biosciences, US]. Cells were permeabilized with Fix Perm and stained for markers and intracellular cytokines with CD69-Texas Red, CD8-APC-H7, IL-2- PE, IFN- $\gamma$-Alexa 700, and TNF- $\alpha$-PE-Cy7 (BD Biosciences, US). Cells were collected on an LSR-II (BD Biosciences, US) and analyzed using a FlowJo device (Treestar, US). All of the data shown have the 'Mock' stimulated background responses subtracted.

\section{Luminex}

Inflamatory cytokines were analyzed in the animal's sera, collected $24 \mathrm{hr}$ post-vaccination. We used The Inflammatory Cytokine Mouse Magnetic 4-Plex Panel for the Luminex ${ }^{\mathrm{mt}}$ (Thermo Fisher Scientific, USA), which detects GM-CSF, IL- $1 \beta$, IL-6, and TNF- $\alpha$. The reads were performed using a Bio-Plex 200 device (Bio-Rad, USA). Three independent experiments were performed.

\section{Statistical analysis}

A one-way ANOVA was used to compare all 5 groups in the experiments. A Tukey-Kramer multiple comparison test was used post-test. Graphics were produced using GraphPad Prism 6.0, and we performed the statistical analyses on GraphPad InStat 3.10.

\section{Results}

We built a DNA vaccine that elicited a VLP in vitro. The rationale was to have the DNA vaccine driving in vivo assembly and budding of a mature Gag VLPs containing BG505 Env, that also carries a portion of the HIV-1 genome. One promoter drives the expression of HIV-1 LTR-GagPro composed of native HIV nucleic acid sequences, which encodes for mature Gag proteins. The RNAm of LTR-GagPro, which will be encapsulated in the particle through Psi sequence in the LTR, can form secondary structures that induce the innate arm of the immune system. The other promoter expresses Rev and Env BG505. Rev protein allows correct HIV RNA processing and packaging [13], and Env protein will be correctly incorporated into budding VLPs as a spike. The contents of these genes were selected to make mature Gag particles that contain Env BG505. BG505 was selected by the Design \& Development Laboratory, International AIDS Vaccine Initiative because it binds to a broad range of Nab [PG9/16, VRC01, PGT122, PGT 125 families] [14]. Mature Gag molecules processed by the HIV protease are more immunogenic than unprocessed Gag polyprotein [Pr55Gag] [15]. Thus the presence of HIV-1 genomic RNA in the VLPs will stimulate the cytosolic innate immunity receptors TLR-7 and RIG-1, which can positively shape and modulate the humoral and cellular responses [16].

In the western blot experiment, we could see that the pVLP-LTRGagPro forms a mature Gag particle due the presence of a p24 band in the membrane. On the other side, LTR-Gag showed only the Pr55Gag band indicating a non-processed Gag, as expected (Fig 2). 


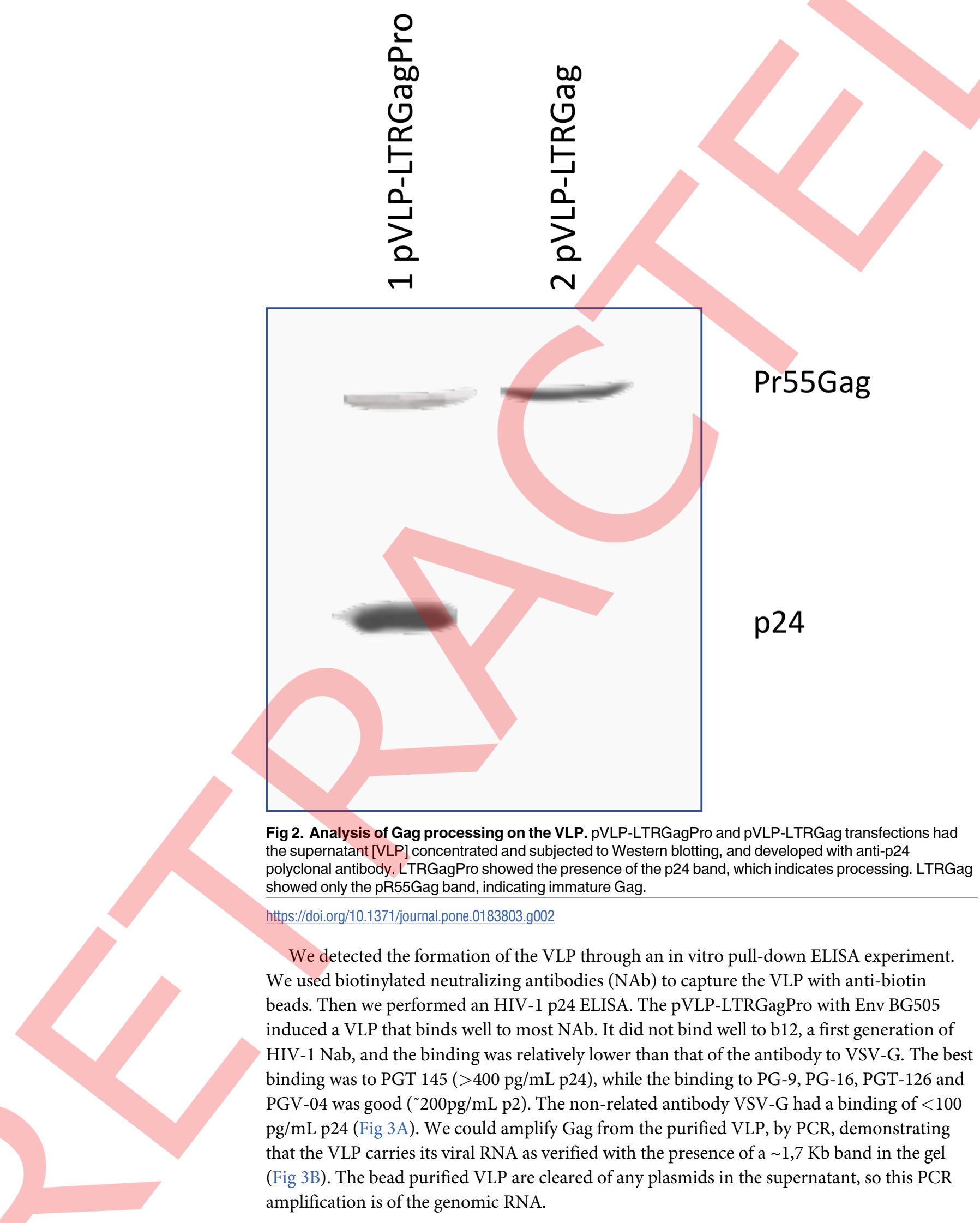



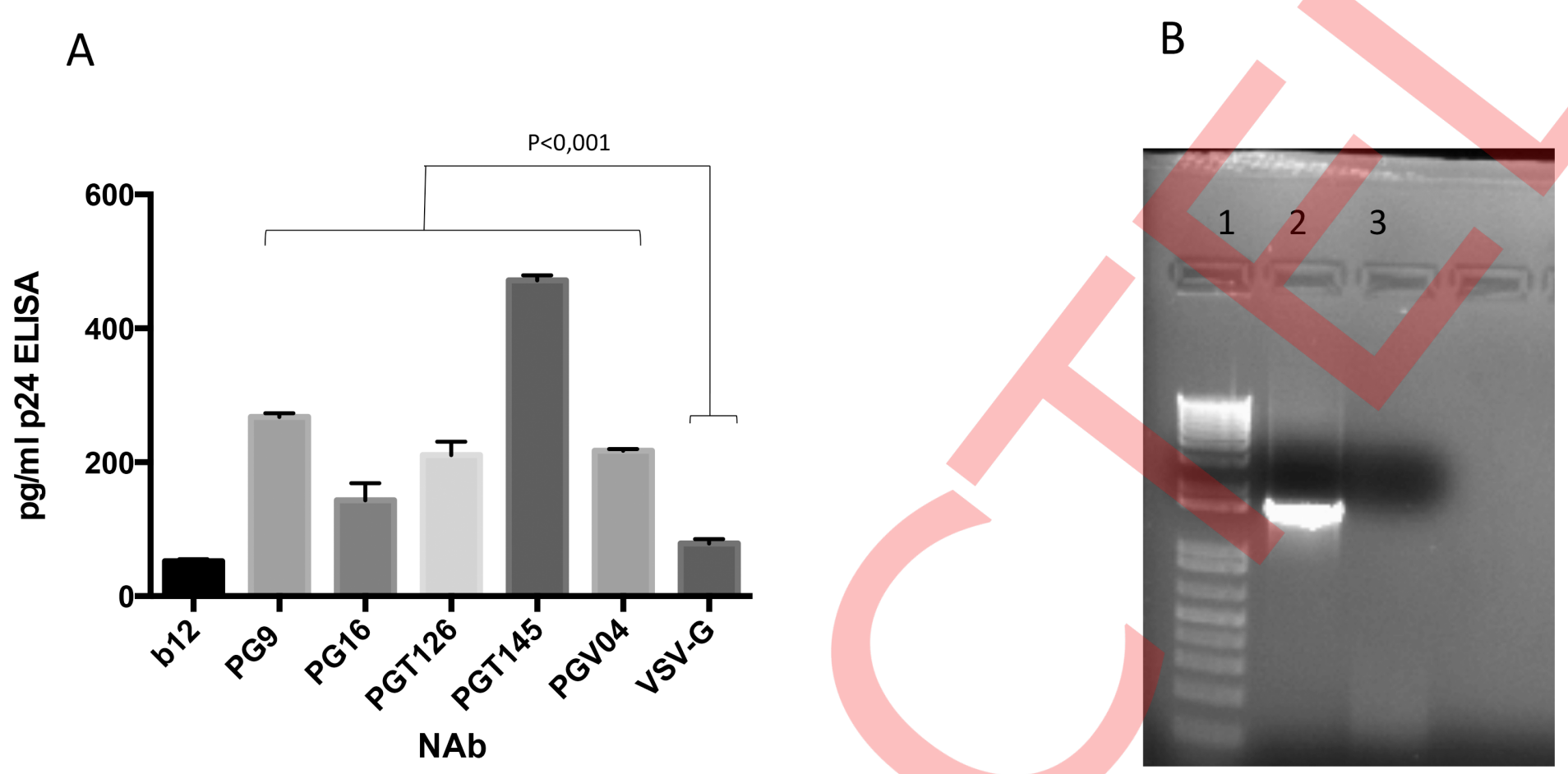

Fig 3. Pull-down of the pVLP-LTRGagPro produced VLP by biotinylated magnetic microbeads. A- after separation of the VLP with the neutralizing antibodies [Nab] [b12, PG9, PG16, PGT126, PGT145, PGV04, and the non-related antibody VSV-G], the purified VLP were submitted to HIV p24 ELISA, proving the correct assembly of the particle. B-the purified VLP RNA was extracted, CDNA was synthetized and PCR amplification was performed for the Gag region [lane 1- $1 \mathrm{~kb}$ ladder plus, lane 2-pVLP-GagPro cDNA, lane 3-negative control].

https://doi.org/10.1371/journal.pone.0183803.g003

On week 5, we could not detect the production of antibodies by pVLP-LTRGagPro. The other constructs showed a moderate level of antibodies production, with a titer of serum dilution 500. Already, on week 9 the pVLP-LTRGagPro was the construct that produced lowest level of antibody [around dilution 200]. pVLP-GagPro produced a higher titer, approximately 2500 serum dilution. pVLP-LTRGag induced a serum dilution of approximately 2000 and pCMV-Gag and-Env, induced a serum dilution of approximately 1000 (Fig 4).

In the ICS experiment we lost the samples from week 5 due a laboratory accident. Thus, we could only analyze the samples from week 9 . From this experiment, we could see that pVLPLTRGagPro induced the highest levels of cytokine production when stimulated with gag pool peptides. For CD4 T-cells, it induced $0.1,0.2$, and $0.35 \%$ of IFN $\gamma$, IL-2, and TNF $\alpha$, respectively (statistically significant). For CD8 T-cells, it produced 0.5, 0.2 and $0.55 \%$ of IFN $\gamma$, IL-2, and $\mathrm{TNF} \alpha$, respectively (statistically significant) (Fig $5 \mathrm{~A}$ and $5 \mathrm{~B}$ ). When comparing the pVLPLTRGagPRO with the regular HIV DNA vaccine, pCMVGag + pCMV-Env, we could detect higher levels of cytokine production in the VLP forming vaccine than in the regular DNA vaccine. For CD4 T-cells, it induced 8.3, 7.0, and 2.2-fold for the cytokines IFN $\gamma$, IL-2, and TNF $\alpha$, respectively. Additionally, it induced 2.5, 12.8, and 2.2-fold for IFN $\gamma$, IL-2, and TNF $\alpha$, respectively in the CD8 T-cells. When stimulated with the gag protein, we could detect statistically significant results for $\mathrm{CD} 4$ cells secreting IFN $\gamma$. When T-cells were stimulated with gp120, we observed another pattern of response. These cells did not express significant levels of cytokines. The pVLP-LTRGag was the only responder. It induced $0.25 \%$ of IFN $\gamma$ for CD8 cells (Fig 5F).

We measured the innate immune response 24 hours after the last vaccine shot. We analyzed the production of the cytokines GM-CSF, IL- $1 \beta$, IL- 6 , and TNF- $\alpha$. For GM-CSF, IL- 6 , and 


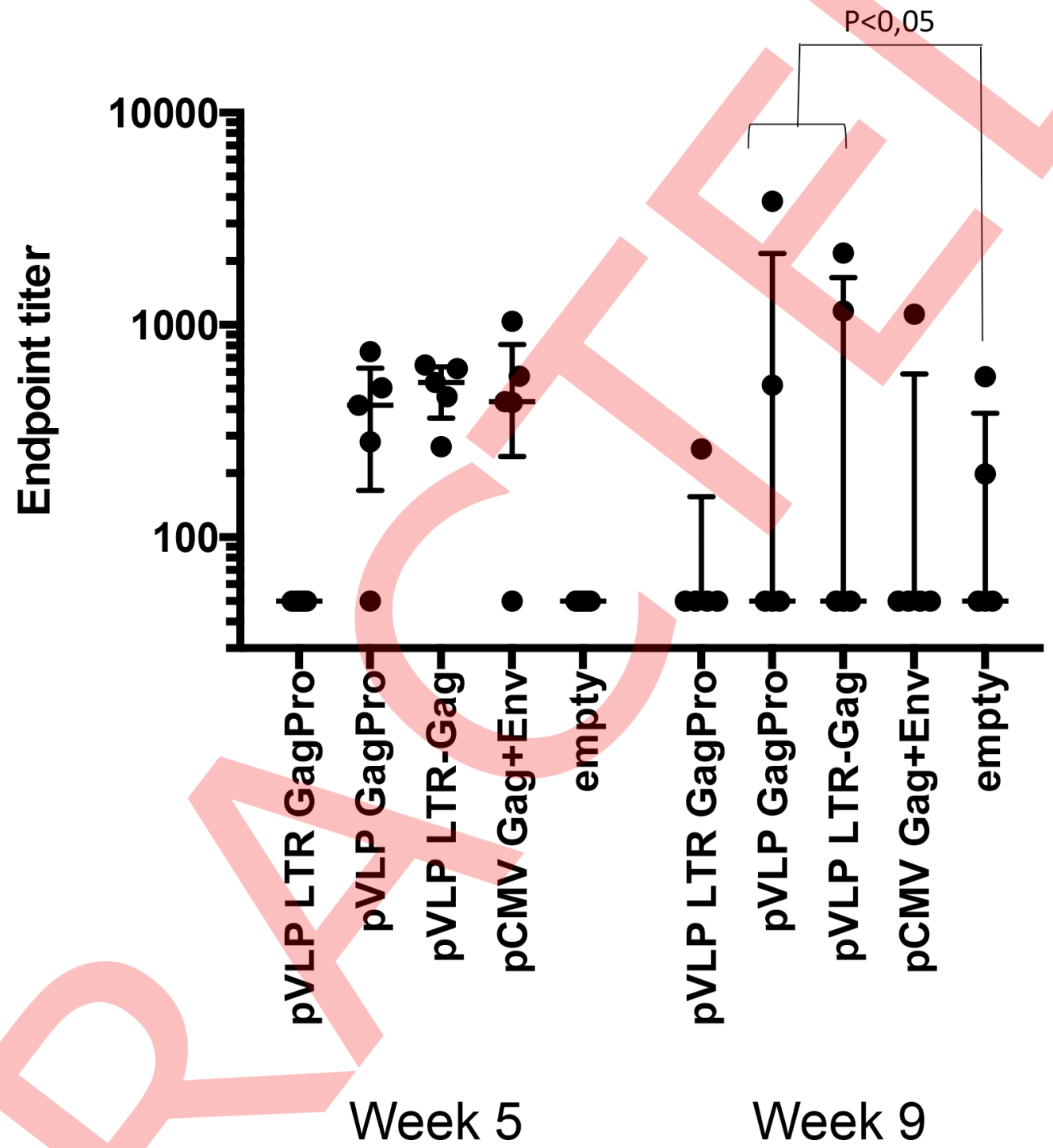

Fig 4. Measurement of the produced antibodies after vaccination by ELISA. The sera of the animals collected on week 5 and 9, were subjected to ELISA, with the Env BG505 coating the plates. Endpoint titers of anti-env antibodies are shown. Statistically significant results are indicated in the figure.

https://doi.org/10.1371/journal.pone.0183803.g004

TNF- $\alpha$ we did not observe differences [data not shown]. We did observe an increase in the production of IL- $1 \beta$ for $\mathrm{pVLP}$-LTRGagPro that was statistically significant, with $\mathrm{p}<0.001$ (Fig 6).

\section{Discussion}

We observed that pVLP-LTRGagPro built a mature particle, with the processed Gag proteins, as shown in the Western blot experiment. pVLP-LTRGag did not show a mature Gag protein, as expected.

In the pull-down experiment, we could see that the VLP (pVLP-LTRGagPro) binds well to all neutralizing antibodies used (VRC-1, PG-9, PG-16, PGT-145, etc). This proved that the VLP was well assembled. Because we detected the capsid (p24) by ELISA, we determined that the BG505 envelope is expressed in the right conformation, thus exposing the CD4 binding 
A

CD4 cells with gag peptides

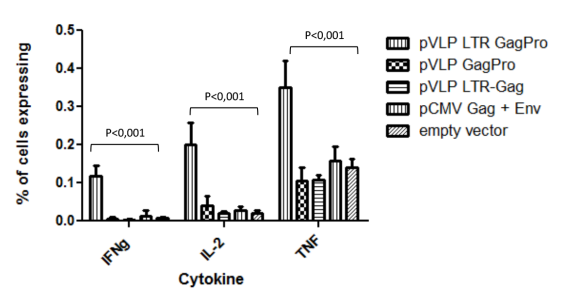

B

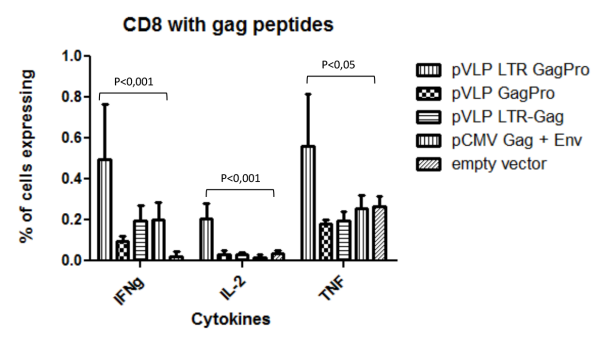

C

CD4 cells with gag protein

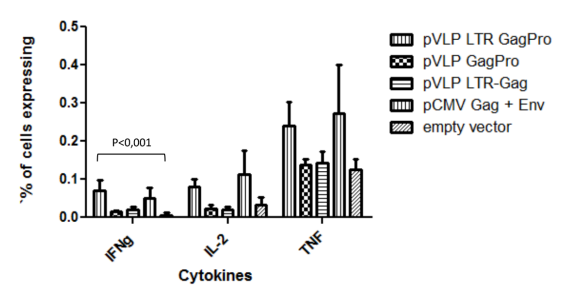

D

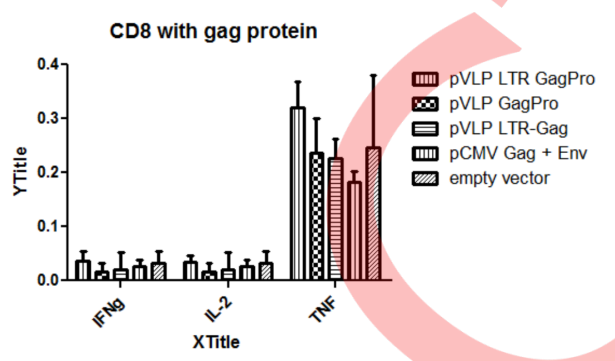

E

CD4 with gp120

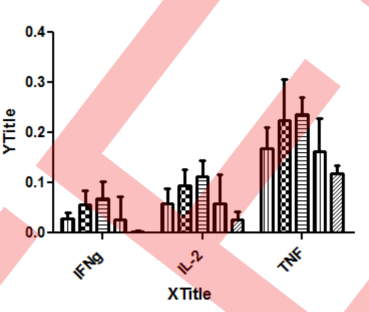

血 pVLP LTR GagPro m p VLP GagPro PVLPLTR-Gag empty vector

Fig 5. Intracellular cytokine staining [ICS] results. CD4 and CD8 T-cells were stimulated with gag peptide pool, gag protein, or Env gp120 protein and the production of the cytokines IFNy, IL-2, and TNFa was measured. The statistically significant results have the $p$ value indicated in the graphics. Samples that do not have $\mathrm{p}$ value shown did not show statistically significant results.

https://doi.org/10.1371/journal.pone.0183803.g005

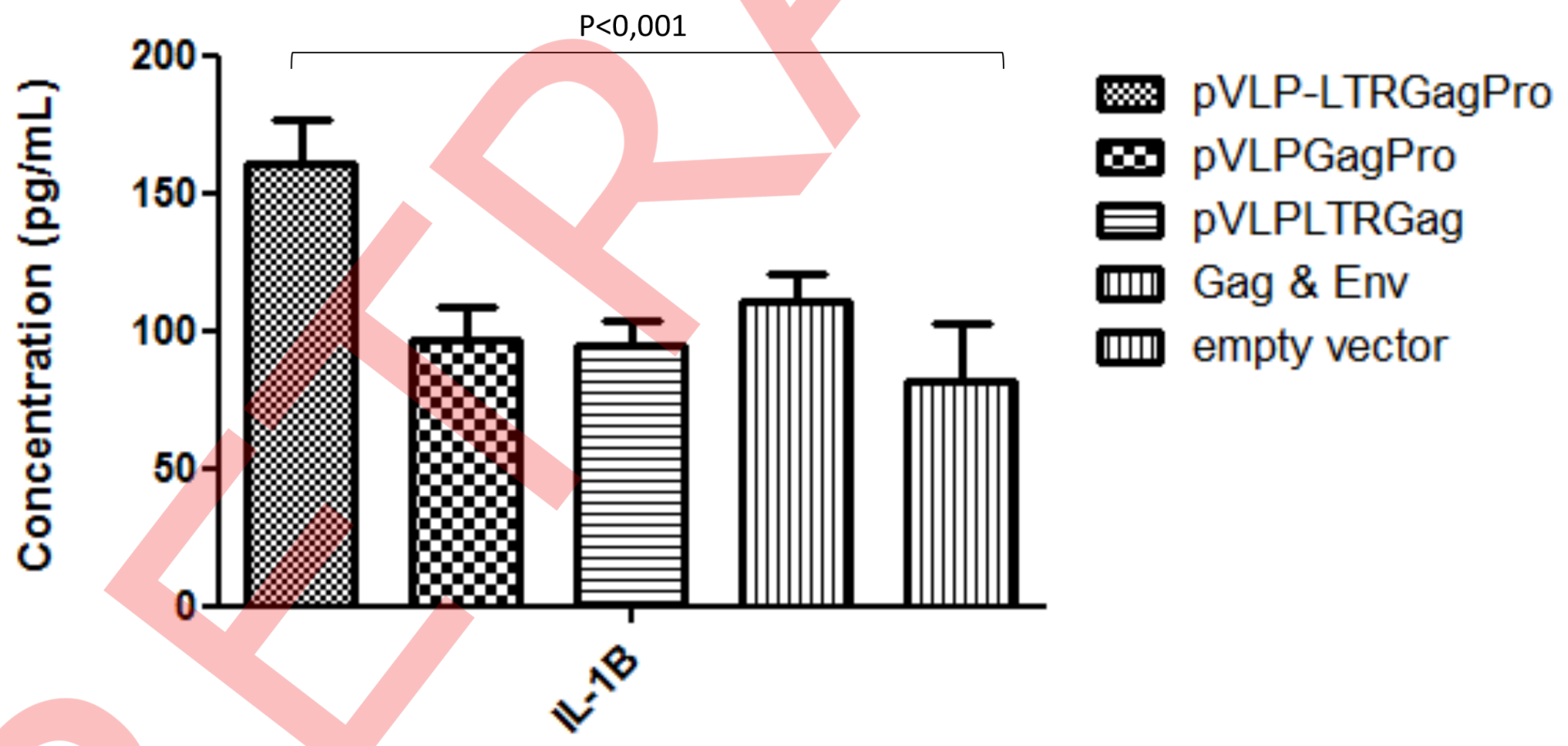

Fig 6. Analysis of the production of pro-inflammatory cytokines, 24 hours post-vaccination. The production of the cytokines GM-CSF, IL-1 $\beta$, IL-6, and TNF- $\alpha$ were measured by Luminex. The cytokines GM-CSF, IL-6, and TNF- $\alpha$ had no significant results [data not shown]. Regarding IL-1 $\beta$, the pVLPLTRGagPro construct induced more liberation of this cytokine than the other groups $[p<0,001]$, for 3 independent experiments.

https://doi.org/10.1371/journal.pone.0183803.g006 
domains, allowing it to bind to the NAbs. Cale et al, also used VLP to stabilize the env trimer and identify HIV-1-neutralizing N90-VRC38 Ab lineage-a good neutralizing antibody [17]. These results confirm the effectiveness of VLPs as presentation and delivery system for conformational proteins [18]. The PCR amplification of Gag from the purified VLP proves that the particle was encapsulating the viral RNA.

Among the cell types analyzed [CD4 e CD8], the pVLP-LTRGagPro showed the highest levels of all produced cytokines [IFN $\gamma$, IL-2 and TNF $\alpha$ ] when pulsed with gag peptides. This construction induced more cytokines than the regular DNA vaccine pCMV-Gag + pCMV-Env. It appears that the response was shifted to a more Th1 pattern. We think that this effect was caused because of the presence of of viral RNA that acted as an adjuvant, triggering the innate immune system. The T-cell responses against Env gp120 was low. This is desirable in a HIV vaccine, as the T-cell response to Env is associated with increasing HIV viremia [6]. The constructs that do not carry RNA were less effective in producing cytokines than the one that carries the RNA.

High magnitude, polyfunctional CD8 T-cell responses to Gag have been shown to be an important component of the control of viremia in HIV positive long term non-progressors and elite controllers. Therefore, Gag is considered to be an important component of HIV vaccines because a strong $\mathrm{T}$ cell response could result in the early clearance of HIV-infected cells via CD8 T-cells at the site of infection, control of the spread from the entry portal, and control of viremia [19].

Chapman et al [20] also made a DNA vaccine for HIV that forms a VLP. However, this VLP was an immature Gag without RNA inside. They had high levels of Gag T-cell responses, similar to ours. However, it is difficult to compare these studies because they used ELISPOT to evaluate the T-cell response and also used only three Gag-specific peptides for the stimulation of the cells. In contrast, we used a Gag peptide pool for stimulation. Another group [21] produced a non-replicative lentivirus DNA vaccine for HIV. It had just one cycle of replication, such as our DNA vaccine. They produced robust Gag T-cell response. Again, it is difficult to compare because they used ELISPOT and a proliferation assay (CFSE) to evaluate the T-cell responses. Fuller et al [22] made a gp120 DNA vaccine and tested it in mice. In contrast to us, they showed high levels of Env T-cell response and moderate antibody response to Env. We found a more robust response than Env antibodies.

The pVLP-LTRGagPro showed lower antibody titers than the others construct. This may be due the presence of the RNA in the VLP acting as adjuvant. Adjuvants can trigger different antibody responses, and they can selectively skew an antibody [23][24]. As the immune response was shifted to a Th1 pattern, the Th2 response was lower, which decreased the production of antibodies in the pVLP-LTRGagPro construct [21].

We tested the innate immune responses 24 hours after vaccination. This was not ideal, and it should have been tested $4-6$ hours post vaccination. Nevertheless, we were able to see a high level of IL-1 $\beta$ upon pVLP-LTRGagPro vaccination, which indicates the activation of the innate immune system.

These results illustrate that VLPs with GagPro provide a safe and effective means of enhancing neutralizing humoral responses to particles containing gp120 proteins. Therefore, the antigenicity of these VLPs represent an attractive approach in the design of vaccines for which specific stimulation of neutralizing antibodies and cytotoxic effector functions to complex glycoproteins is desired [25].

\section{Conclusion}

The vaccine tested here, pVLP-LTRGagPro, showed better immunogenic results than its controls. It showed higher ICS results for both $\mathrm{CD} 4$ and $\mathrm{CD} 8 \mathrm{t}$-cells. The high Gag response of the 
T-cell and the low Env T-cell response is desirable for an HIV vaccine [6]. This vaccine produced more IL-1 $\beta 24$ hours post-vaccination than others groups, indicating that this vaccine can activate the innate immune system. We demonstrated that this vaccine has its own natural adjuvant. This construct not only induced antibodies but also caused the immune cellular response to shift to a more favorable Th1 profile. Thus, this vaccine induced all of the arms of the immune system: cellular and humoral responses as well as the activation of the innate immune system.

The next step for this vaccine should be testing in NHP [non-human primates]. In the NHP setting, we could evaluate non-innate immune response and determine whether this vaccine is able to protect animals from infection upon challenge from an SHIV model.

\section{Acknowledgments}

We would like to thank Michael Caulfield and Christopher Parks for scientific discussion and support.

\section{Author Contributions}

Conceptualization: Alexandre Calazans, Cesar Boggiano.

Data curation: Alexandre Calazans, Cesar Boggiano.

Formal analysis: Alexandre Calazans, Cesar Boggiano, Ross Lindsay.

Investigation: Alexandre Calazans, Cesar Boggiano, Ross Lindsay.

Methodology: Alexandre Calazans, Cesar Boggiano, Ross Lindsay.

Project administration: Alexandre Calazans, Cesar Boggiano.

Resources: Alexandre Calazans, Ross Lindsay.

Software: Alexandre Calazans, Cesar Boggiano.

Supervision: Alexandre Calazans, Cesar Boggiano.

Validation: Alexandre Calazans, Cesar Boggiano, Ross Lindsay.

Visualization: Alexandre Calazans, Ross Lindsay.

Writing - original draft: Alexandre Calazans, Cesar Boggiano, Ross Lindsay.

Writing - review \& editing: Alexandre Calazans, Cesar Boggiano, Ross Lindsay.

\section{References}

1. Barré-Sinoussi F, Chermann JC, Rey F, Nugeyre MT, Chamaret S, Gruest J, Dauguet et al. 1983. Isolation of a T-lymphotropic retrovirus from a patient at risk for acquired immune deficiency syndrome [AIDS]. Science [80-] 220:868-871. PMID: 6189183

2. Kuscetti FW, Gallagher KE, Clarkson B, Kalyanaraman VS, Harbor CS, I M, Svoboda J et al. 1984. Frequent Detection and Isolation of Cytopathic Retroviruses [HTLV-III fron Patients with AIDS and at Risk for AIDS. Science [80-] 224:500-503. PMID: 6200936

3. Excler JL, Robb ML, Kim JH. 2015. Prospects for a globally effective HIV-1 vaccine. Vaccine 33:D4D12. https://doi.org/10.1016/j.vaccine.2015.03.059 PMID: 26100921

4. Barouch DH. 2008. Challenges in the development of an HIV-1 vaccine. Nature 455:613-619. https:// doi.org/10.1038/nature07352 PMID: 18833271

5. McDermott AB, Koup RA. 2012. CD8[+] T cells in preventing HIV infection and disease. AIDS 26:1281-92. https://doi.org/10.1097/QAD.0b013e328353bcaf PMID: 22441256 
6. Kiepiela P, Ngumbela K, Thobakgale C, Ramduth D, Honeyborne I, Moodley E et al. 2007. CD8+ T-cell responses to different HIV proteins have discordant associations with viral load. Nat Med 13:46-53. https://doi.org/10.1038/nm1520 PMID: 17173051

7. Liao H-X, Lynch R, Zhou T, Gao F, Alam SM, Boyd SD et al. 2013. Co-evolution of a broadly neutralizing HIV-1 antibody and founder virus: supplement. Nature 496:469-76. https://doi.org/10.1038/ nature12053 PMID: 23552890

8. Rolland M, Edlefsen PT, Larsen BB, Tovanabutra S, Sanders-Buell E, Hertz T et al. 2012. Increased HIV-1 vaccine efficacy against viruses with genetic signatures in Env V2. Nature 490:417-20. https:// doi.org/10.1038/nature11519 PMID: 22960785

9. Levitz SM, Golenbock DT. 2012. Beyond empiricism: Informing vaccine development through innate immunity research. Cell 148:1284-1292. https://doi.org/10.1016/j.cell.2012.02.012 PMID: 22424235

10. Pulendran B, Ahmed R. 2011. Immunological mechanisms of vaccination. Nat Immunol 12:509-17. PMID: 21739679

11. Liu MA. 2010. Immunologic basis of vaccine vectors. Immunity 33:504-515. https://doi.org/10.1016/j. immuni.2010.10.004 PMID: 21029961

12. Hoffenberg S, Powell R, Carpov A, Wagner D, Wilson A, Kosakovsky Pond S et al. 2013. Identification of an HIV-1 clade A envelope that exhibits broad antigenicity and neutralization sensitivity and elicits antibodies targeting three distinct epitopes. J Virol 87:5372-83. https://doi.org/10.1128/JVI.02827-12 PMID: 23468492

13. Lu K, Heng X, Garyu L, Monti S, Garcia EL, Kharytonchyk S et al. 2011. NMR detection of structures in the HIV-1 5'-leader RNA that regulate genome packaging. Science 334:242-5. https://doi.org/10.1126/ science.1210460 PMID: 21998393

14. Julien J-P, Lee JH, Cupo A, Murin CD, Derking R, Hoffenberg S et al. 2013. Asymmetric recognition of the HIV-1 trimer by broadly neutralizing antibody PG9. Proc Natl Acad Sci U S A 110:4351-6. https:// doi.org/10.1073/pnas.1217537110 PMID: 23426631

15. Sacha JB, Reynolds MR, Buechler MB, Chung C, Jonas AK, Wallace LT et al. 2008. Differential antigen presentation kinetics of CD8+ T-cell epitopes derived from the same viral protein. J Virol 82:9293-8. https://doi.org/10.1128/JVI.00749-08 PMID: 18596093

16. Heil F, Hemmi H, Hochrein H, Ampenberger F, Kirschning C, Akira S et al. 2012. Species-Specific Recognition of Single-Stranded RNA via Toll-like Receptor 7 and 8. Science [80-] 1526.

17. Cale Evan M., Gorman Jason, Radakovich Nathan A., Kwong Peter D., Mascola John R. et al.2017. Virus-like Particles Identify an HIV V1V2 Apex-Binding Neutralizing Antibody that Lacks a Protruding Loop. Immunity 46, 777-791. https://doi.org/10.1016/j.immuni.2017.04.011 PMID: 28514685

18. Visciano ML, Diomede L, Tagliamonte M, Tornesello ML, Asti V, Bomsel M et al. 2011. Generation of HIV-1 Virus-Like Particles expressing different HIV-1 glycoproteins. Vaccine. 2011 Jul 12; 29[31]:490312. https://doi.org/10.1016/j.vaccine.2011.05.005 PMID: 21596074

19. Williamson AL, Rybicki EP. 2015. Justification for the inclusion of Gag in HIV vaccine candidates. Expert Rev Vaccines 15:1-14. https://doi.org/10.1586/14760584.2016.1112744

20. Chapman R, Jongwe TI, Douglass N, Chege G, Williamson A-L. 2017. Heterologous prime-boost vaccination with DNA and MVA vaccines, expressing HIV-1 subtype $C$ mosaic Gag virus-like particles, is highly immunogenic in mice. PLoS One 12:e0173352. https://doi.org/10.1371/journal.pone.0173352 PMID: 28278263

21. Moussa M, Arrode-Bruss G, Manoylov I, Malogolovkin A, Mompelat D, Ishimwe $\mathrm{H}$ et al. 2015. A novel non-integrative single-cycle chimeric HIV lentivector DNA vaccine. Vaccine 33:2273-2282. https://doi. org/10.1016/j.vaccine.2015.03.021 PMID: 25825333

22. Fuller D, Haynes J. 1994. A qualitative progression in HIV type 1 glycoprotein 120-specific cytotoxic cellular and humoral immune responses in mice receiving a DNA-based glycoprotein 120 vaccine. AIDS Res Hum Retroviruses 10:1433-41. https://doi.org/10.1089/aid.1994.10.1433 PMID: 7888198

23. Gunn BM, Alter G. 2016. Modulating Antibody Functionality in Infectious Disease and Vaccination. Trends Mol Med 22:969-982. https://doi.org/10.1016/j.molmed.2016.09.002 PMID: 27756530

24. Vaccari M, Gordon SN, Fourati S, Schifanella L, Liyanage NPM, Cameron M et al. 2016. Adjuvantdependent innate and adaptive immune signatures of risk of SIVmac251 acquisition. Nat Med 22.

25. Deml Ludwig, Schirmbeck Reinhold, Reimann Jörg, Wolf Hans, and Wagner Ralf. 1997. Recombinant Human Immunodeficiency Pr55gag Virus-like Particles Presenting Chimeric Envelope Glycoproteins Induce Cytotoxic T-Cells and Neutralizing Antibodies. Virology 235, 26-39. https://doi.org/10.1006/ viro.1997.8668 PMID: 9300034 